patients that received adjuvant therapy and $12.4 \%$ of the entire cohort. We compared the 3 groups: no adjuvant therapy (NAT), adjuvant therapy with classical criteria (ATW) and adjuvant therapy without fulfilling classical criteria (ATWO). Disease-free survival (DFS) at 4.5 years was $90.4 \%$ (89.291.6\%), 85.2\% (83.2-87.2\%), and 91.7\% (89.3-94.1\%) respectively. No difference in DFS was observed after adjustment for previous conization, tumour size $>2 \mathrm{~cm}$, and minimally invasive approach.

Conclusion* In this European cohort, a higher proportion of patients who received adjuvant treatment was observed in comparison with the literature in women with early cervical cancer after radical hysterectomy. However, no differences in DFS were observed between groups.

\section{INCIDENCE OF LYMPH NODE METASTASIS IN CERVICAL CARCINOMA WITH $\leq 5$ MM DEPTH OF INVASION AND >7 MM HORIZONTAL SPREAD}

1;2 L Nicolai ${ }^{*},{ }^{3} \mathrm{R}$ Yigit, ${ }^{4} \mathrm{MCG}$ Bleeker, ${ }^{3} \mathrm{~J}$ Bart, ${ }^{4} \mathrm{~J}$ Van der Velden, ${ }^{2} \mathrm{CH}$ Mom. ${ }^{1}$ University Medical Center Groningen, Gynecology, Groningen, Netherlands; ${ }^{2}$ Amsterdam UMC, locatie AMC, Gynecology, Amsterdam, Netherlands; ${ }^{3}$ University Medical Center Groningen, Pathology, Groningen, Netherlands; ${ }^{4}$ Amsterdam UMC, locatie AMC, Pathology, Amsterdam, Netherlands

\subsection{6/ijgc-2021-ESGO.35}

Introduction/Background* According to the 2018 International Federation of Gynecology and Obstetrics (FIGO) staging system, cervical cancer with $\leq 5 \mathrm{~mm}$ depth of invasion (DOI) and $>7 \mathrm{~mm}$ horizontal spread, first classified as FIGO stage IB, are now classified as stage IA. For this group of cervical cancers, it is unclear what the risk of lymph node metastases (LNM) is, and consequently, what treatment is recommended. This study aims to determine the incidence of LNM in patients with tumors confined to the cervix, with $\leq 5 \mathrm{~mm}$ DOI and with $>7 \mathrm{~mm}$ horizontal spread, and to study the association between histological type, diameter, lymph-vascular space invasion (LVSI) and LNM in this group.

Methodology In this retrospective study, we selected all women diagnosed with FIGO (2009) IB cervical cancer between 1985 and 2020, with a tumor with $\leq 5 \mathrm{~mm}$ DOI and $>7 \mathrm{~mm}$ diameter from patient records of the Amsterdam University Medical Center (Amsterdam UMC) and the University Medical Center Groningen (UMCG). All cases with LNM were revised by an expert pathologist. The incidence of LNM was calculated with $95 \%$ confidence interval (CI) for the whole population. The associations between histological type, DOI, diameter and LVSI with LNM were evaluated by calculating odds ratios (OR) with 95\% confidence intervals (CI) using logistic regression.

Result(s)* Of the 398 patients included, 16 had pathologically confirmed LNM (4\%, 95\% CI 2.3\% - 6.4\%). No difference in LNM incidence was found between histological types. The incidence of LNM in our cohort was not significantly increased in the presence of LVSI, OR 3.61 (95\% CI 0.97 7.4). More LNM were seen in patients with a tumor diameter of $\geq 20 \mathrm{~mm}$ compared to the group of patients with a tumor $<20 \mathrm{~mm}$, OR 5.0 (95\% CI: 1.81-13.82, $\mathrm{p}=.002$ ). No cases with LNM were found in the tumors with a DOI of $\leq 3 \mathrm{~mm}$ without LVSI.

Conclusion* Lymph node assessment is recommended for patients in 2018 FIGO stage IA with a diameter $>7 \mathrm{~mm}$, because of a $4 \%$ incidence of LNM. Since no LNM were found in the subgroup of patients in FIGO stage IA1 with a diameter $>7 \mathrm{~mm}$ without LVSI, lymph node assessment is not recommended for this specific group.

\section{EFFECT ON OVERALL SURVIVAL OF ADYUVANT CHEMOTHERAPY IN LOCALLY ADVANCED CERVICAL CANCER (LACC)}

AF Rave Ramirez, D González García-Cano*, M Laseca Modrego, O Arencibia Sanchez, A Martín Martínez. Complejo Hospitalario Universitario Insular Materno Infantil de Gran Canaria, Gynecologic Oncology, las palmas de gran canaria, Spain

\subsection{6/ijgc-2021-ESG0.36}

Introduction/Background* The objective of the study was to determine whether the addition of adjuvant chemotherapy to chemoradiotherapy improves overall survival in patients with locally advanced cervical cancer (LACC).

Methodology Retrospective observational cohort study. We included patients with diagnosis of LACC, (Stages IIIA, IIIB, IIIC1, IIIC2 or IVA according to FIGO 2018), who received adjuvant chemotherapy (carboplatin and paclitaxel), during the period of study from 2013 to 2018 (n: 35), comparing it with a control group (n: 38).

Result(s)* 73 patients were included in the study from which 35 received adjuvant treatment with chemotherapy. The survival rate at 3 and 5 years was $77.1 \%$ and $68.6 \%$ for the group who received adjuvant and $10.5 \%$ and $7.9 \%$ for those who did not. ( $p<0.001, \mathrm{p}<0.001$, respectively). Gastrointestinal, genitourinary, and neuropathic toxicities were observed during chemotherapy, but only $3.1 \%$ and $0 \%$ were grade 3 or 4 respectively. The most severe toxicites were haematologic, in terms of neutropenia (G3/4: 33.4\%) and anemia (G3/4: $33.4 \%)$. Only 5 patients $(14,3 \%)$ needed to suspend chemotherapy treatment.

Conclusion* Adjuvant chemotherapy in patients with LACC significantly improved survival disease-free and overall survival with acceptable toxicity percentages. Prospective trials are needed to confirm these findings.

\section{VALIDATION OF STRUCTURAL AND PROCESS ESGO QUALITY INDICATORS FOR SURGICAL TREATMENT OF CERVICAL CANCER IN A LARGE EUROPEAN COHORT}

${ }^{1 ; 2}{ }^{2}$ Boria* ${ }^{2} E$ Chacon, ${ }^{2} \mathrm{~N}$ Manzour, ${ }^{3} \mathrm{~N}$ Martin, ${ }^{2} \mathrm{D}$ Vazquez, ${ }^{2} \mathrm{~T}$ Castellano, ${ }^{2} \mathrm{JA}$ Mínguez, ${ }^{2} \mathrm{JL}$ Alcazar, ${ }^{4} \mathrm{~T}$ Iscar, ${ }^{5 ; 6} \mathrm{~A}$ Gonzalez-Martin, ${ }^{6} \mathrm{~L}$ Sánchez Lorenzo, ${ }^{6} \mathrm{~J}$ Espinos, ${ }^{7} \mathrm{M}$ Cambeiro, ${ }^{2}$ LM Chiva. ${ }^{1}$ Clinica universidad de navarra, Madrid, Spain; ${ }^{2}$ Clinica universidad de navarra, Gynecologic Oncology, Madrid, Spain; ${ }^{3}$ Clinica universidad de navarra, Statistics; ${ }^{4}$ Clinica universidad de navarra, Pathology; ${ }^{5}$ Clinica universidad de navarra, Oncologia; ${ }^{6}$ Clinica universidad de navarra, Oncology; ${ }^{7}$ Clinica universidad de navarra, Radiotherapic oncology

\subsection{6/ijgc-2021-ESG0.37}

Introduction/Background* Implementation of quality surgical care programs as a component of comprehensive multi-disciplinary management has been shown to improve outcomes in patients with colorectal cancer and other types of malignancies. $^{1,2}$ In that scenario, the ESGO quality indicators for surgical treatment of cervical cancer were published. ${ }^{3}$

The aim of this study was to validate quality indicators for surgical treatment of cervical cancer in a large European retrospective cohort and to analyze if its accomplishment may impact the disease-free survival (DFS) in patients with cervical cancer. 


\section{Abstract 488 Table 1}

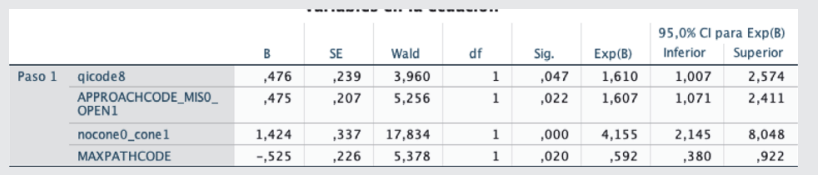

Abstract 488 Table 2
\begin{tabular}{|l|r|r}
\hline & Frecuencia & Porcentaje \\
\hline 5 & 12 & 1,4 \\
\hline 6 & 29 & 3,3 \\
\hline 7 & 97 & 11,1 \\
\hline 8 & 321 & 36,6 \\
\hline 9 & 344 & 39,2 \\
\hline 10 & 74 & 8,4 \\
\hline Total & 877 & 100,0 \\
\hline
\end{tabular}

Methodology The succor database was fulfilled in 2019 for the succor study ${ }^{4}$, comprising a total of of 1272 patients who underwent radical hysterectomy for IB1 cervical cancer in Europe between 2013 and 2014. In March 2021 a survey was conducted among all the Succor participating centers. The questionnaire included questions regarding the accomplishment of the ESGO process and structural quality indicators, comprising a total of 9 items with a maximum punctuation of 10 .

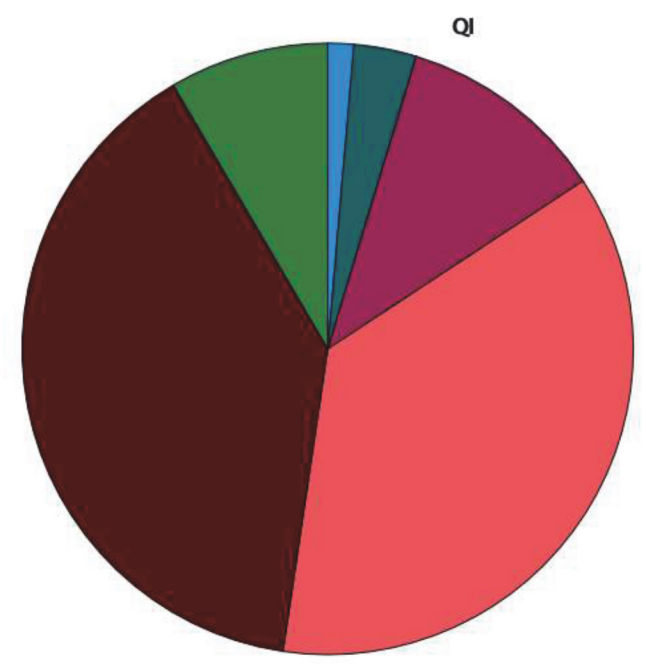

Abstract 488 Figure 2

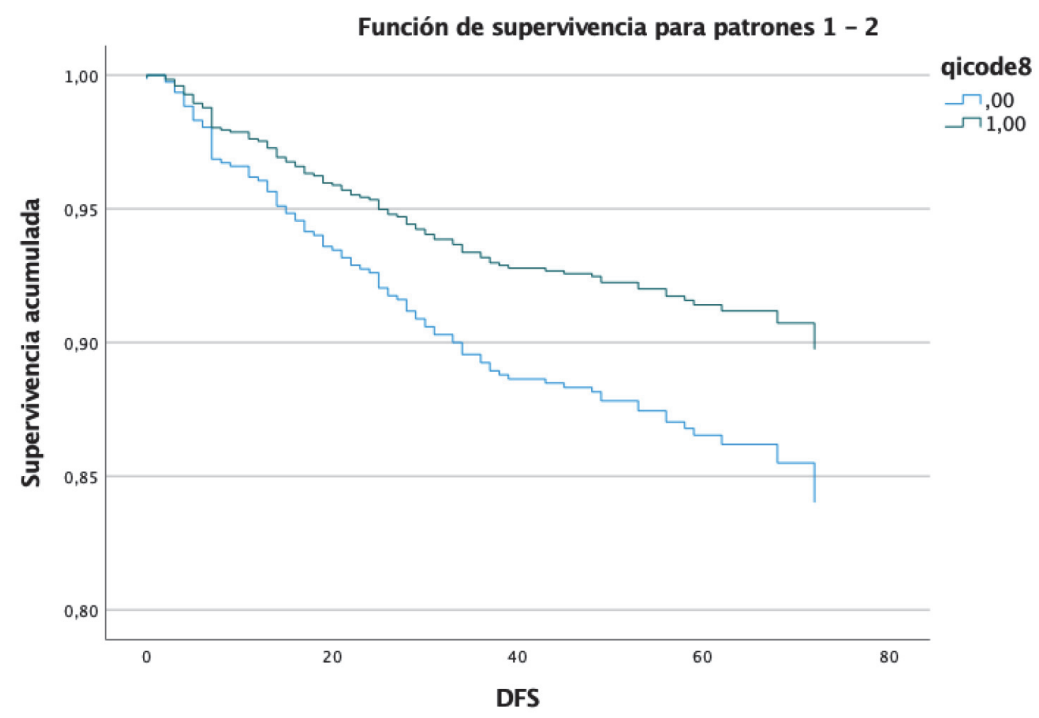

\begin{tabular}{|c|c|c|c|c|c|c|c|c|c|}
\hline & & & & & & \multirow[b]{3}{*}{ Sig. } & \multirow[b]{3}{*}{$\operatorname{Exp}(B)$} & \multirow{2}{*}{\multicolumn{2}{|c|}{$95,0 \%$ Cl para $\operatorname{Exp}(B)$}} \\
\hline & & & & & & & & & \\
\hline & & B & SE & Wald & df & & & Inferior & Superior \\
\hline \multirow[t]{4}{*}{ Paso 1} & qicode 8 & ,476 & ,239 & 3,960 & 1 & ,047 & 1,610 & 1,007 & 2,574 \\
\hline & $\begin{array}{l}\text { APPROACHCODE_MISO_ } \\
\text { OPEN1 }\end{array}$ & ,475 & ,207 & 5,256 & 1 & ,022 & 1,607 & 1,071 & 2,411 \\
\hline & nocone0_cone 1 & 1,424 & ,337 & 17,834 & 1 & , 000 & 4,155 & 2,145 & 8,048 \\
\hline & MAXPATHCODE &,- 525 &, 226 & 5,378 & 1 &, 020 & ,592 & 380 & ,922 \\
\hline
\end{tabular}

Abstract 488 Figure 1 
Potential factors that may influence on the relapse risk in this cohort were previously analyzed. ${ }^{51} \mathrm{~A}$ regression cox analysis including all these factors and quality indicators accomplishment was conducted. Good accomplishment of QI was defined as accomplishment of 8 or more of them.

Result(s)* A total of 82 out of the 126 participating institutions answered the survey. The final cohort was composed of 877 patients. Percentages of accomplishment of quality indicators are showed in table 1 . Two groups were defined according to the number of quality indicators accomplished (Group 1: 7 or less vs group 2: 8 or more). The rate of DFS at 4.5 year for patients who underwent surgery in centers that accomplished 7 or less quality indicators was $82.1 \%$ (78.8$85.6)$ and $89.9 \%(88.7-91.1 \%)$ in the other group. Low accomplishment of QI was associated with a lower rate of disease-free survival than High accomplishment of QI (HR, 1.61; 95\% CI, 1.01 to $2.57 ; \mathrm{P}=0.047)$. A difference that remained after adjustment for previous conization, tumour size $>2 \mathrm{~cm}$, and minimally invasive approach.

Conclusion* Good accomplishment of structural and process QI was associated with a better DFS in this European cohort.

\section{CHALLENGES IN DIAGNOSIS OF CERVICAL ADENOCARCINOMA IN A PATIENT WITH UTERINE MALFORMATION: A RARE CASE REPORT}

\footnotetext{
1;2S Álvarez Sánchez, 1;2JM Barreiro García, 1;2M Marti Sopeña, 1;2JJ Delgado Espeja, 1;2JA Solano Calvo, ${ }^{1 ; 2}$ Á Zapico Goñi. ${ }^{1}$ Hospital Universitario Príncipe de Asturias, Gynaecology, Alcalá de Henares, Spain; ${ }^{2}$ Facultad de Medicina, Universidad Alcalá de Henares, Alcalá de Henares, Spain
}

\subsection{6/ijgc-2021-ESG0.38}

Introduction/Background* Cervical cancer is the fourth most common cancer of women in the world. Some risk factors for cervical adenocarcinoma are shared with squamous cell carcinoma, mainly infection with high-risk subtypes of human papillomavirus (HPV).

Methodology A 69-year-old woman suffered recurrent postmenopausal bleeding for 2 years. Medical and surgical history: a right radical nephrectomy due to congenital renal atrophy and a partial removal of a rudimentary uterus during a c-section.

Initial medical research showed no pathology, including double liquid-based cytologies with negative result to HPV. During the follow-up period, a solid excrecent mass was discovered protruding into vagina.

Result(s)* The vaginal biopsy diagnosed a papilar adenocarcinoma of clear-cells. A computed tomography and nuclear magnetic resonance revealed a $5 \mathrm{~cm}$ solid mass located in the right sided genital system. A hysteroscopic study was also performed and revealed no lesions in vagina but a double cervix and uterus. The left-sided hemiuterus presented an atrophic endometrium, and the right-sided hemiuterus showed a proliferative endometrium. The endometrial biopsy revealed also a papilar adenocarcinoma of clear-cells with immunohistochemical study that suggested the cervical origin.

The interdisciplinary tumour committee considered the case as a cervical cancer stage IIIB, performing the paraaortic lymphadenectomy by laparoscopic surgery (negative for malignancy) and subsequently, chemoradiotherapy.

Conclusion* The difficulty of diagnosis in cervical adenocarcinoma lies in the cytology; the lack of sensitivity to adenocarcinoma may be due to these cancers developing in the endocervical canal, making sampling of abnormal cells difficult.

Clear-cell carcinoma comprises approximately $3 \%$ of all cervical adenocarcinomas and is aetiologically unrelated to HPV infection.

This case highlights some points of technical difficulties to resolve the diagnosis. First, the genital malformation that created obstacles to diagnose the origin of the pathology. Secondly, the negative result to HPV. Lastly, the unusual subtype of cervical adenocarcinoma and its predicament connected with the diagnostic sensitivity in the cytology.

\section{VARIABLES THAT PREDICT NODAL STATUS OF PATIENTS WITH EARLY CERVICAL CANCER IN THE SUCCOR COHORT}

A Berasaluce, N Martín-Calvo, E Chacon, F Boria, N Manzour, LM Chiva*. University of Navarra

\subsection{6/ijgc-2021-ESGO.39}

Introduction/Background* We aimed to identify the variables associated with higher odds of positive nodes in women in the SUCCOR study and describe both disease free survival and overall survival by nodal status in those women.

Methodology We used data from the SUCCOR study, a European multicentre study that collected retrospective information of 1272 women who underwent a radical hysterectomy by open or minimally invasive surgery for stage IB1 cervical cancer (FIGO 2009) between January 2013 and December 2014 After exclusions, the final sample included 1157 patients. Missing values were imputed with the median in quantitative variable and grouped in a new category in qualitative ones. We compared sociodemographic and clinical characteristics of patients by node status. We used Student's t test for quantitative variables and Pearson's chi squared test for qualitative ones. Variables with a $\mathrm{p}$ value below 0.05 in the univariate analyses were introduced in a logistic regression model with stepwise forward selection. Finally, we compared disease free survival and overall survival by nodal status using the LogRank test

Result(s)* We found that women with large tumours (>2 $\mathrm{cm})$, linfovascular space invasion, parametrial invasion, postoperative complications or positive (or $<2 \mathrm{~mm}$ free) margins in the tumour were more likely to have positive nodes In the multivariable adjusted model, the variables that independently predict higher odds of positive nodes were: large tumour size (OR: 1.8; 95\% CI:1.18-2.78), linfovascular space invasion (OR: 5,34; 95\% CI 3.31-8.61), parametrial invasion (OR: 5.10; 95\% CI 2.23-11.67), positive margins in the tumour (OR: 2,44; 95\% CI 1.18-5.06), and postoperative complications (OR: 2.22; 95\% CI: 1.42-3.48). The survival analyses showed significant differences in both disease-free survival $(p=0.007)$ and overall survival $(p=0,011)$ between groups.

Conclusion* In the Succor cohort the presence of large tumour size, lymphovascular space invasion, parametrial invasion, positive margins in the tumour and postoperative complications were associated with the presence of positive lymph nodes and worse survival. 